\title{
Psychiatric sequelae in women who experience life- threatening complications during pregnancy in a low-and-middle income country (LMIC)
}

Nadira Khamker ( $\nabla$ nadira.khamnker@up.ac.za )

University of Pretoria https://orcid.org/0000-0003-1349-6909

Johannes Lodewikus Roos

University of Pretoria

Research article

Keywords: LMIC, developing countries, life threatening complications, delivery, psychiatric sequelae

Posted Date: February 25th, 2020

DOI: https://doi.org/10.21203/rs.2.24517/v1

License: (c) (i) This work is licensed under a Creative Commons Attribution 4.0 International License.

Read Full License 


\section{Abstract}

Purpose: Researchers compared psychiatric sequelae in women with life threatening complications during pregnancy (LT), and uncomplicated pregnancies (UP) in a low- and middle-income country (LMIC) over a 6month follow-up period.

Method: The study design was a mixed method and quantitative data was collected in a parallel convergent manner at two university hospitals in Gauteng, South Africa. Women from two groups, namely those with uncomplicated pregnancies and those with life threatening complications were followed up over a six-month period postnatally.

Results: Eighty-nine women participated in the study of whom 46 experienced a life-threatening pregnancy complication. At six-week follow-up, $65.5 \%(n=19)$ of women in the LT-group and $29.2 \%(n=7)$ of the UPgroup experienced symptoms of depression $(p=0.08)$. In the LT-group, $30.4 \%(n=14)$ had anxiety symptoms compared to $7 \%(n=3)$ of UP-group $(p=0.011)$. Compared to the UP-group, more women in the LT-group experienced somatic symptoms $(28.3 \%, n=13, p=0.003)$ and sleep disturbances $(28.3 \%, n=13, p=0.0009)$. More women in the LT-group reported memory disturbance $15.2 \%(n=7, p=0.034)$ compared to women in the UP-group. Psychotic symptoms were not prominent in either group.

Conclusions: Women who experienced life-threatening complications at birth experienced significant distress including depression, anxiety, somatic complaints, sleep disturbances and memory deficits compared to women with uncomplicated pregnancies and deliveries. Maternal services for women with complicated deliveries should include a comprehensive package of obstetric, paediatric and psychiatric care that involves a multi-professional team.

\section{Introduction}

Research linking women's reproductive and mental health is an area of increasing focus (Chandra and Ranjan 2007). Pregnancy related complications are the leading cause of death and disability worldwide among women between the ages of 15 and 44 . Obstetric complications are common in low-income countries. (Ronsmans, 2009) where there is a high risk of maternal death for up to six months postpartum. (Ronsmans et al, 2006). Women who experience severe life-threatening complications are at a greater risk for developing psychiatric complications and have poorer health outcomes than women with uncomplicated pregnancies (Patel, 2008). However, studies that describe the experiences of women who survive life-threatening complications are scarce (Kaaja et al, 2010; Weeks et al, 2005), especially in developing or low-income settings.

A systematic review revealed that maternal mental disorders are approximately three times more prevalent in low- and middle-income countries (LMIC) than in high income countries (HIC). (Fisher et al, 2010)

South African women in poor urban areas have to rely on public health care provided at primary levels of care. Despite the improvements and utilisation of antenatal care services, an increase in perinatal mortality rates persists. (Bekinska et al, 2008) Postnatal care for women in South Africa focuses primarily on the 
infant with limited attention paid to maternal health, including maternal mental health. (Chopra et al, 2009) High prevalence rates of depressed mood among pregnant and post-natal women have been reported in community based epidemiological studies in South Africa. (Honikman et al, 2012) Up to 40\% of mothers in poor environments have depression, usually untreated, exacerbated by intimate partner violence, lack of resources, higher levels of poverty, HIV and unwanted pregnancy (Kathree et al. 2014, Marsay et al, 2017) Despite the high levels of depression during the perinatal period, there is a lack of mental health care services, (Nyatsanza et al. 2016) with no routine screening or treatment of mental health during the perinatal period in the busy public health settings . (Honikman et al, 2012; Marsay et al. 2017).

Psychiatric disorders that occur during the perinatal period are associated not only with morbidity and mortality in the women affected, but also with increased morbidity in their children. Exposure to maternal psychiatric illness may represent a stressful life event and increase the child's vulnerability to future psychopathology. (Stuart et al, 1998)

In South Africa, the importance of treating perinatal psychiatric conditions is just coming to light, thus studies that identify target groups for treating these conditions are particularly important.

Fillipi et al (2010) concluded that women with complicated births would benefit from active screening for symptoms and signs of adverse emotional and physical wellbeing during postnatal visits or other contacts with health professionals during the postpartum period. The researchers explored whether women who experience life threatening complications (LT) were more vulnerable to developing psychiatric complication, compared to women who had uncomplicated deliveries (UP). If so, did women in the LT group experience more severe and long-term psychiatric sequelae?

\section{Method}

The study was conducted at two university hospitals affiliated in Gauteng, South Africa. These hospitals serve the greater area of the City of Tshwane, which has approximately 1.98 million inhabitants, and an average population growth of $1.7 \%$. Patients who are admitted to university or tertiary level hospitals are either self-referrals or referred from surrounding maternal obstetric units, district hospitals and surrounding provinces.

Patients with uncomplicated deliveries (UP) were selected randomly from the birth registers of the respective labour wards of the two hospitals. The women with life-threatening complications (LT) were selected if they met the criteria for a "near-miss" pregnancy. A "near-miss" pregnancy is defined by the WHO is "a severe life-threatening obstetric complication that requires urgent medical intervention to prevent the death of the mother" (WHO 2004:106). The near-miss condition is defined according to clinical, laboratory based and management-based criteria (Table 1).

Participants were recruited in collaboration with colleagues from the Department of Obstetrics at the two hospitals. Near miss cases are identified during the daily routine audit by a team of consultants, registrars and medical officers in the department. Prospective participants were approached soon after delivery. Participants with life-threatening complications who were severely ill after delivery were approached when 
they were medically stable. The researcher discussed the purpose of the study with prospective participants and distributed patient information leaflets. Where women had difficulties with language, nursing staff served as interpreters during the informed consent process. Participants received a copy of the informed consent once they agreed to participate.

The researcher interviewed participants at four-time intervals. During these interviews the researcher completed questionnaires, symptom measures and scales. The four-time intervals were, shortly after the delivery, at six weeks, at three months and at six months postpartum. Initially, the researcher conducted a comprehensive psychiatric interview with each participant, and obtained a medical and obstetric history. The researcher recorded socio-demographic information including age, race, level of education, employment status and current living circumstances for each participant.

The participants completed two crosscutting symptom measures, both which have been validated. Language interpreter support was used where necessary. The Level 1 cross-cutting symptom measure broadly identified the presence of the following psychiatric symptoms: depression, anxiety, sleep, cognition, dissociation, self-harm, anger, psychosis, mania, post-traumatic stress, obsessive-compulsive and substance abuse. Subsequent to scoring and interpretation of the Level 1 findings, the researcher completed a Level 2 crosscutting symptom measure for patients with psychiatric symptoms. The Level 2 crosscutting symptom measure is a Patient-Reported Outcomes Measurement Information System (PROMIS) Scale, which measure significant-symptoms of psychiatric illness. The PROMIS Scales used in this study included symptoms for depression, anxiety, mania, substance abuse, repetitive thoughts and behaviour, anger, cognition, somatic and sleep disturbances.

Once the researcher completed the Level $1 \& 2$ measures, appropriate validated psychiatric rating scales were completed, including the Beck Depression Inventory and Hamilton Anxiety Scale. The Beck Depression Inventory assessed the intensity of depression. This scale has been validated for use in obstetric populations (cut-off 16) (Holcomb et al, 1996). Additionally, the researcher used the WHO Disability Assessment to measure impairments in personal, social and occupational functioning. Data were analysed using the SSPE software programme.

This study was approved by the Ethics Committee at the University of Pretoria (353/2013), the Doctoral Protocol Committee and the chief executive officers at the two hospitals.

\section{Results}

Eighty-nine women signed informed consent to participate in the study. Of these, 43 were women with uneventful pregnancies and 46 had LT deliveries (Table 1). The study participants varied in age from $16-$ 45 years, with almost a fifth of them being older than 36 years. Approximately a third of the women $(n=15)$ in the life-threatening (LT) group experienced an intrauterine foetal demise.

A summary of the psychiatric sequelae identified at each time interval is presented in table 2 and notable psychiatric sequelae are elaborated on. 
Table 1: Demographic information of mothers who experienced life threatening (LT) complications and uncomplicated pregnancies (UP) at 2 hospitals in the Tshwane region, Gauteng, South Africa

\begin{tabular}{|c|c|c|c|}
\hline Age distribution & UP n (\%) & LT n (\%) & Total (\%) \\
\hline $16-20$ & $7(16.3 \%)$ & $6(13.3 \%)$ & $13(14.8 \%)$ \\
\hline $21-25$ & $10(23.3 \%)$ & $11(24.4 \%)$ & $21(23.9 \%)$ \\
\hline $26-30$ & $10(23.3 \%)$ & $11(24.4 \%)$ & $2(23.9 \%)$ \\
\hline $31-35$ & $8(18.6 \%)$ & $9(20.0 \%)$ & 17 (19.3\%) \\
\hline $36-40$ & $6(14.0 \%)$ & $7(15.6 \%)$ & $13(14.8 \%)$ \\
\hline $41-45$ & $2(4.7 \%)$ & $1(2.2 \%)$ & $3(3.4 \%)$ \\
\hline \multicolumn{4}{|l|}{ Population group } \\
\hline Black & 37 (86.0\%) & $44(95.7 \%)$ & $81(91.0 \%)$ \\
\hline Mixed race & $0(0.0 \%)$ & $1(2.2 \%)$ & $1(1.1 \%)$ \\
\hline Asian/Indian & $0(0.0 \%)$ & $1(2.2 \%)$ & $1(1.1 \%$ \\
\hline White & $6(14.0 \%)$ & $0(0.0 \%)$ & $6(6.7 \%)$ \\
\hline \multicolumn{4}{|l|}{ Relationship status } \\
\hline Married & 13 (30.2\%) & 16 (34.8\%) & 29 (32.6\%) \\
\hline Single & 13 (30.2\%) & 15 (32.6\%) & $28(31.5 \%)$ \\
\hline Living together & 15 (34.9\%) & $8(17.4 \%)$ & $23(25.8 \%)$ \\
\hline Non-exclusive relation & $2(4.7 \%)$ & 7 (15.2\%) & $9(10.1 \%)$ \\
\hline \multicolumn{4}{|l|}{ Employment status } \\
\hline Working full-time & $12(27.9 \%)$ & $17(37.0 \%)$ & 29 (32.6\%) \\
\hline Working part-time & $6(14.0 \%)$ & $7(15.2 \%)$ & $13(14.6 \%)$ \\
\hline Not-working (housewife) & $6(14.0 \%)$ & $5(10.9 \%)$ & $11(12.4 \%)$ \\
\hline Student/Scholar & $5(11.6 \%)$ & $3(6.5 \%)$ & $8(9.0 \%)$ \\
\hline Unemployed & $14(32.6 \%)$ & $14(30.4 \%)$ & $28(31.5 \%)$ \\
\hline \multicolumn{4}{|l|}{ Living circumstances } \\
\hline Standalone house/structure & $20(46.5 \%)$ & $20(43.5 \%)$ & $40(44.9 \%)$ \\
\hline Flat in block of flats & $5(11.6 \%)$ & $5(10.9 \%)$ & $10(11.2 \%)$ \\
\hline House/flat/room in backyard & $6(14.0 \%)$ & $5(10.9 \%)$ & $11(12.4 \%)$ \\
\hline Informal dwelling/shack & 12 (27.9\%) & $16(34.8 \%)$ & $28(31.5 \%)$ \\
\hline
\end{tabular}

Table 2:Summary of psychiatric sequelae observed in women who experience life threatening (LT) complications and uncomplicated pregnancies (UP). Assessments were conducted just after delivery (P1), at six weeks (P2), at three months (P3) and at six months (P4). 


\begin{tabular}{|c|c|c|c|c|c|c|c|c|c|c|c|c|}
\hline Condition & P1 & & & $\mathrm{P} 2$ & & & P3 & & & $\mathrm{P} 4$ & & \\
\hline & LT (46) & $\mathrm{U}(43)$ & $\begin{array}{l}\mathrm{p}- \\
\text { value }\end{array}$ & LT (29) & $\mathrm{U}(24)$ & $\begin{array}{l}\mathrm{p}- \\
\text { value }\end{array}$ & LT (21) & $\mathrm{U}(11)$ & $\begin{array}{l}\mathrm{p}- \\
\text { value }\end{array}$ & $\mathrm{LT}(12)$ & U (8) & $\begin{array}{l}\mathrm{p}- \\
\text { value }\end{array}$ \\
\hline Depression & $\begin{array}{l}14 \\
(30.4 \%)\end{array}$ & $\begin{array}{l}8 \\
(18.6 \%)\end{array}$ & 0,196 & $\begin{array}{l}19 \\
(65.5 \%)\end{array}$ & $\begin{array}{l}7 \\
(29.2 \%)\end{array}$ & 0,008 & $\begin{array}{l}10( \\
(47.6 \%)\end{array}$ & $\begin{array}{l}2 \\
(18,2 \%)\end{array}$ & 0,102 & $\begin{array}{l}2 \\
(16.7 \%)\end{array}$ & $\begin{array}{l}1 \\
(12.5 \%)\end{array}$ & 0,798 \\
\hline Mania & $\begin{array}{l}1 \\
(2.2 \%)\end{array}$ & $\begin{array}{l}1 \\
(2.3 \%)\end{array}$ & 0,962 & $\begin{array}{l}1 \\
(3.4 \%)\end{array}$ & $\begin{array}{l}2 \\
(8.3 \%)\end{array}$ & 0,444 & 0 & 0 & & 0 & 0 & \\
\hline Anxiety & $\begin{array}{l}14 \\
(30.4 \%)\end{array}$ & $\begin{array}{l}3 \\
(7.0 \%)\end{array}$ & 0,005 & $\begin{array}{l}16 \\
(55.2 \%)\end{array}$ & $\begin{array}{l}5 \\
(20.8 \%)\end{array}$ & 0,011 & $\begin{array}{l}9 \\
(42.9 \%)\end{array}$ & $\begin{array}{l}2 \\
(18.2 \%)\end{array}$ & 0,163 & $\begin{array}{l}1 \\
(8.3 \%)\end{array}$ & $\begin{array}{l}1 \\
(12.5 \%)\end{array}$ & 0,761 \\
\hline Anger & $\begin{array}{l}2 \\
(4.3 \%)\end{array}$ & $\begin{array}{l}1 \\
(2.3 \%)\end{array}$ & 0,597 & $\begin{array}{l}5 \\
(17.2 \%)\end{array}$ & $\begin{array}{l}1 \\
(4.2 \%)\end{array}$ & 0,135 & $\begin{array}{l}3 \\
(14.3 \%)\end{array}$ & 0 & 0,188 & 0 & 0 & \\
\hline Somatic & $\begin{array}{l}13 \\
(28.3 \%)\end{array}$ & $\begin{array}{l}2 \\
(4.7 \%)\end{array}$ & 0,003 & $\begin{array}{l}11 \\
(37.9 \%)\end{array}$ & $\begin{array}{l}3 \\
(12.5 \%)\end{array}$ & 0,037 & $\begin{array}{l}1 \\
(4.8 \%)\end{array}$ & $\begin{array}{l}1 \\
(9.1 \%)\end{array}$ & 0,631 & 0 & 0 & \\
\hline Sleep & $\begin{array}{l}13 \\
(28.3 \%)\end{array}$ & $\begin{array}{l}3 \\
(7.0 \%)\end{array}$ & 0,009 & $\begin{array}{l}18 \\
(62.1 \%)\end{array}$ & $\begin{array}{l}6 \\
(25.0 \%)\end{array}$ & 0,007 & $\begin{array}{l}8 \\
(38.1 \%)\end{array}$ & $\begin{array}{l}2 \\
(18.2 \%)\end{array}$ & 0,248 & $\begin{array}{l}1 \\
(8.3 \%)\end{array}$ & 0 & 0,402 \\
\hline Memory & $\begin{array}{l}7 \\
(15.2 \%)\end{array}$ & $\begin{array}{l}1 \\
(2.3 \%)\end{array}$ & 0,034 & $\begin{array}{l}10 \\
(34.5 \%)\end{array}$ & $\begin{array}{l}2 \\
(8.3 \%)\end{array}$ & 0,024 & $\begin{array}{l}5 \\
(23.8 \%)\end{array}$ & $\begin{array}{l}1 \\
(9.1 \%)\end{array}$ & 0,311 & 0 & 0 & \\
\hline Psychosis & $\begin{array}{l}3 \\
(6.5 \%) \\
\end{array}$ & $\begin{array}{l}1 \\
(2.3 \%)\end{array}$ & 0,34 & $\begin{array}{l}2 \\
(6.9) \\
\end{array}$ & $\begin{array}{l}1 \\
(4.2 \%)\end{array}$ & 0,669 & 0 & $\begin{array}{l}1 \\
(9.1 \%)\end{array}$ & 0,16 & 0 & 0 & \\
\hline Substance & $\begin{array}{l}1 \\
(2.2 \%)\end{array}$ & $\begin{array}{l}2 \\
(4.7 \%)\end{array}$ & 0,518 & $\begin{array}{l}1 \\
(3.4 \%)\end{array}$ & $\begin{array}{l}3 \\
(12.5 \%)\end{array}$ & 0,214 & $\begin{array}{l}1 \\
(4.8 \%)\end{array}$ & 0 & 0,462 & 0 & 0 & \\
\hline
\end{tabular}

\section{Depressive symptoms}

At six weeks, almost two-thirds $(65.5 \%, \mathrm{n}=19)$ of women in the LT-group presented with depressive symptoms, compared to women in the UP-group $(29.2 \%, n=7)(p=0.08$, OR 4.91). At three months, fewer women experienced depressive symptoms, but more women $(47.6 \%, n=10)$ in the LT-group still presented with depressive symptoms, compared to women in the UP-group (18.2\%, $n=2)(p=0.102$, OR 4.09). At six months, $16.7 \%$, $(n=2)$ of women in the LT-group and $12.5 \%(n=1)$ of the UP-group continued to experience residual depressive symptoms $(p=0.798$, OR 1.40$)$.

At delivery, 12 (13.5\%) women had Becks depression (BDI) scores of more than 18 indicating moderate to severe depression. Of these, 9 (75\%) women had LT deliveries and 3 (25\%) had UP deliveries. At six weeks, 29 women $(62.1 \%)$ of women in the LT group presented with BDI scores of more than 18 . At the threemonths, 11 women (23.8\%) in the LT-group had a BDI score of more than 18, while at 6 months only one participant had a BDI score of more than 18.

\section{Anxiety symptoms}


At the first assessment, just after delivery, 14 (30.4\%) of women in the LT-group presented with anxiety symptoms, compared to $3(7 \%)$ women in the UP-group ( $p=0.005$, OR 5.83). More women presented with anxiety symptoms at the six-week assessment, remaining more prevalent in the LT-group $(n=16,55.2 \%)$ compared to the UP-group $(n=5,20.8 \%)(p=0,011, O R 4.6)$. Fewer women experienced anxiety symptoms at three months (LT: $n=9,42.9 \%$; UP: $n=2,18.2 \%, p=0,163$, OR 3.38). At six months, only one woman in each group presented with residual symptoms of anxiety. At delivery, $17.6 \%(n=16)$ of women had moderate to severe symptoms of anxiety with HAM-A scores of greater than 18 . At six weeks, 32.1\% $(n=29)$ women had HAM-A scores of greater than 18 , and of these, $75 \%(n=22)$ were women who had LT deliveries. Of these 22 , only $10(36.4 \%)$ women presented with anxiety symptoms at three months.

\section{Somatic symptoms}

Participants also presented with mild to moderate somatic symptoms (Table 2). At the first assessment, more women in the LT-group $(n=13,28.3 \%)$ presented with somatic symptoms, compared with women in the UP-group $(n=2,4.7 \%)(p=0.003,0 R 8.08)$. This difference was maintained at six weeks (LT: $n=11$, $37.9 \%$; UP: $n=3,12.5 \%, p=0.037$, OR 4,28).

\section{Sleep disturbances}

At the first assessment, more women in the LT-group $(n=13,28.3 \%)$ reported sleep disturbances compared to women in the UP-group $(n=3,7.0 \%)(p=0.009$, OR 5.25 ,). More women from both groups reported sleep disturbances at the six-week interview (LT: $n=18,62 \%$; UP: $n=6,25 \%, p=0.007,0 R 4.91)$. Sleep disturbances improved over time.

\section{Cognitive and memory disturbances}

At the first assessment, more women in the LT-group $(n=7,15.2 \%)$ reported memory disturbances compared to women in the UP-group $(n=1,2.3 \%)(p=0.034$, OR 7.54,). At six weeks, this difference intensified with, $34.5 \%(n=10)$ and $8.3 \%(n=2)$ in LT-group and UP-group respectively presenting with memory disturbances ( $p=0.024$, OR 5.79).

\section{Psychotic symptoms:}

At the first assessment, $6.5 \%(n=3)$ of women in the LT-group and $2.3 \%(n=1)$ of women in the UP-group experienced psychotic symptoms ( $p=0.340$, OR 2.93). At delivery, $4.5 \%(n=4)$ women presented with a Brief psychiatric rating score of more than 31 , indicating moderate to marked illness. At six weeks and at three months, $3.7 \%(n=2)$ and $3.1 \%(n=1)$ of participants, respectively, were moderately ill with a BPRS score of 38.

\section{Discussion}

Findings from this study indicate the psychiatric sequelae are more common in women who experience life threatening complications at delivery. We identified that women who experienced life threatening 
complications were more prone to depression, anxiety, somatic symptoms, sleep complaints and memory/cognitive deficits while psychotic, manic and substance abuse appeared on the lower symptom domain. These sequelae persisted up to six months following birth, but most conditions resolved over the medium term. Where mothers presented with psychotic symptoms directly after birth, symptoms seemed to persist over the medium term.

\section{Depression}

In this study, few women in both groups presented with symptoms of depression at the first assessment after delivery. These figures increased markedly at the six weeks follow up visit, with more women in the LT group showing symptoms of depression. Whilst only $10 \%(n=4)$ of women experiencing LT births showed symptoms of severe depression (BDI>16) directly after birth, this proportion rose to $65.5 \%(n=19)$ at six weeks. In this study, women with moderate to severe depressive symptoms were referred to a local psychiatric clinic for further management, which may account for fewer women experiencing symptoms of depression at three- and six-month follow-up visits.

The high rates of depression observed at six weeks may be linked to bereavement in women who had experienced foetal loss, but these women presented with additional symptoms that suggested depressive syndrome. These women experienced neuro-vegetative symptom changes, cognitive and memory deficits and impairment in functioning. In the clinical situation, the overlap between bereavement and major depressive disorder may be difficult to elucidate, and such cases should be closely monitored.

Depressive symptoms are common in both traumatic and uncomplicated deliveries. Usually depressive symptoms steadily decrease over time during the peri-partum period (Rahman and Creed, 2010; O'Hara et al, 1984; Campbell and Cohn, 1997; Najman et al, 2000). In developing countries, the prevalence of postpartum depression ranges between 16 and 39\% (Sword et al, 2011; Gonidakis et al, 2008), which is in line with our findings for women with UP deliveries. Our finding suggests that women who experience LT complications during delivery are prone to developing symptoms of depression.

\section{Anxiety}

Anxiety symptoms showed a similar trend to the afore-mentioned depressive symptoms during the fourtime intervals, ranging between $8 \%$ and $55 \%$ in the group of women who experienced LT deliveries. Anxiety symptoms may form part of the depressive syndrome. Globally, the prevalence of perinatal anxiety disorders range between 15 and 39\% (Mota et al, 2008), but few studies have assessed the prevalence of anxiety disorders among women who experience life-threatening medical complications during delivery (Fairbrother et al, 2017).

Anxiety related diagnoses may include acute stress disorder, post-traumatic stress disorder and adjustment disorder with comorbid mood and anxiety symptoms. Women who experience severe medical or obstetric complications to the extent that their well-being and lives are threatened, may present with severe anxiety symptoms indicative of acute stress disorder. In this study, women, followed up at sixmonths, did not present with symptoms of reliving, intrusive thoughts, and nightmares that are suggestive 
of post-traumatic stress disorder. Deliveries complicated by traumatic events, including preeclampsia, has been linked to PTSD or depression (Gaugler-Senden et al, 2012). Diagnosing PTSD requires long term follow-up which was beyond the scope of the study. Additionally, all mothers were closely followed up at six weeks, and referred for care if necessary, possibly pre-empting more serious psychiatric sequelae. Mothers from Brazil who experienced near-miss pregnancies, also did not fulfil the criteria for PTSD, rather they were diagnosed with acute stress disorder which resolved during the postpartum period (Souza et al, 2009).

In this study, the researchers considered adjustment disorder with depressed mood and anxiety symptoms for women presenting with self-limiting anxiety symptoms during the follow-up period. Initially, women in the LT-group experienced greater variation in their ability to function but became less functionally impaired during the three- and six-month follow-up.

\section{Somatic symptoms}

The LT-group presented with significant somatic symptoms after birth (28.3\%), which reached a peak at six weeks and decreased at three months. The somatic symptoms could have been a part of the symptom complex associated with severe illness at the time of delivery

Persisting somatic symptoms, including sleep disturbances could be manifestations of underlying depressive disorder. Women who are depressed may express their distress through somatic symptoms, even during pregnancy (Duer, Schwenk, Coyne,1988; Simon et al, 1999). In Zimbabwe, women with depression are known to express their somatic symptoms (Abas and Broadhead, 1997), and in Ethiopia, women were more likely to have somatic symptoms if they had depression or anxiety after delivering their babies (Senturk et al, 2012). In this study, women presented simultaneously with depression and somatic symptoms at delivery and at six-week follow-up, which may indicate atypical postpartum depression. Sleep disturbances, like somatic symptoms, can occur in pregnancy and the postpartum period due to physiological changes. These processes are required to re-establish homeostasis, but persistent somatic disturbances and inadequate sleep may exacerbate feelings of depression and anxiety. Our findings support the importance of somatisation of mental distress during the perinatal period.

\section{Cognitive and memory deficits}

Similar to somatic symptoms and sleep disturbances, cognitive and memory deficits, at birth and after six weeks, were associated with mothers who experienced LT deliveries. The rates of memory deficits described in this study fall within the rates described in the literature (Porcels et al, 2013). Cognitive and memory deficits at birth may be associated with complications and interventions during hospital admission. Women with eclampsia and preeclampsia are prone to vascular dysfunction and clotting abnormalities as well as cerebral oedema, which could have contributed to memory deficits (Postma et al, 2010). In this study, a quarter of the participants were HIV-positive, which may have contributed to cognitive deficits.

\section{Psychosis}


In this study, four women presented with psychotic symptoms at birth, with two women having persistent symptoms at six weeks and three months. In previous studies, the incidence of postpartum psychosis is found to be one or two per thousand births (Munk-Olsen et al, 2006; Kendell et al, 1987). In this study, women presenting with psychotic symptoms, could have been delirious, which could have resolved with prompt treatment of underlying medical complications.

\section{Limitations of the study}

The findings in this study need to be interpreted with care, since the sample was small, there was a high attrition rate, recall bias and linguistic barriers. In the South African public health sector, follow-up appointments for normal deliveries and for non-South African citizens are difficult leading to loss of participants over time. Where recall bias from participants were problematic, all efforts were made to obtain this information from the clinical files. Nursing staff was used where linguistic barriers occurred.

\section{Conclusion}

In this study, women who experienced life-threatening experiences during birth experienced significant psychiatric distress at the time of birth and during follow-up. Psychiatric domains of pathology identified included: depression, anxiety, somatic complaints, sleeps disturbance and memory/cognitive deficits. The findings add to limited research done in LMIC on women who experience life-threatening complications at birth. Elucidating the different psychiatric presentations into diagnostic categories may be of help in the bio psychosocial management of these cases. It is important for maternal services to include a comprehensive package of care that not only includes members of multidisciplinary teams including obstetricians, paediatricians but management of these women should include appropriate referral to psychiatric services with involvement of a multi-professional team.

\section{Declarations}

Ethical approval and consent to participate: "All procedures performed in studies involving human participants were in accordance with the ethical standards of the institutional and/or national research committee and with the 1964 Helsinki declaration and its later amendments or comparable ethical standards."

This study was approved by the Ethics Committee at the University of Pretoria (353/2013), the Doctoral Protocol Committee and the chief executive officers at the two hospitals.

Informed consent: Informed consent was obtained from all individual participants included in the study.

\section{Consent for publication}

Not applicable

\section{Availability of data and materials}


The datasets used and/or analysed during this study are available from the corresponding author on reasonable request

\section{Conflict of Interest:}

\section{Author 1, declares that she has no conflict of interest. Author 2, receives incentive funding as a rated researcher from the National Research Foundation.}

Funding: No funding was obtained for conducting this study.

\section{Authors' contributions}

All authors contributed to the manuscript. All authors read and approved the final manuscript

\section{Acknowledgements:}

Authors would like to thank Professor RC Pattinson and colleagues from the Department of Obstetrics and Gynecology, Steve Biko Academic Hospital and Kalafong Hospital, Pretoria, South Africa

\section{References}

- Abas, M.A., Broadhead, J.C. 1997. Depression and anxiety among women in an urban setting in Zimbabwe. Psychological Medicine. 27(1):59-71.

- Campbell, S.B., Cohn, J.F. 1997. The timing and chronicity of postpartum depression: implications for infant development. In: Cooper, P.J. editor. Postpartum depression and child development. New York: Guilford. p. 165-97.

- Chandra, P.S., Ranjan, S. 2007. Psychosomatic obstetrics and gynecology- a neglected field? Current Opinion in Psychiatry. 20:168-73.

- Chopra, M., Lawn, J.E., Sanders, D., Barron ,P. Abdool Karim, S.S. et al. 2009. Saving the lives of south Africa's mothers, babies and children: can the health system deliver? Lancet. 374: 835-846.

- Duer, S., Schwenk, T.L., Coyne, J.C. Medical and psychosocial correlates of self-reported depressive symptoms in family practice. Journal of Family Practice. 27(6):609 - 14.

- Fairbrother, N., Young, A.H., Zhang, A., Janssen P., Atony, M.M. 2017. The prevalence and incidence of perinatal anxiety disorders among women experiencing a medically complicated pregnancy. Archives of Women's Mental Health. 20:311-9.

- Fillippi, V., Ganaba, R., Baggaley, R.F., Marshall, T., Storeng, K.T., Sombie, I., et al. 2007. Health of women after severe obstetric complications in Burkina Faso: a longitudinal study. Lancet. 370:132937.

- Fillippi, V., Goufodji, S., Sisimanidis, C., Kanhonou, L., Fottrell, E., Ronsmans, C., et al. 2010. Effects of severe obstetric complications on women's health and infant mortality in Benin. Tropical Medicine and International Health. 15(6):733-42. 
- Fisher J, Mello C.D, Patel V., Rahman A., Tran T., et al. (0212) Prevalence and determinants of common perinatal mental disorders in women in low-and lower-middle income countries: a systematic review. B World Health Organ 90: 139-149.

- Gaugler-Senden, I.P.M., Duivenvoorden, H., Filius, A., De Groot, C.J.M., Steegers, E.A.P., Passchier, J. 2012. Maternal psychosocial outcome after early onset preeclampsia and preterm birth. Journal of Maternal Fetal and Neonatal Medicine. 25(3):272-6.

- Gonidakis, F., Rabavilas, A.D., Varsou, E., Kreatsa, G., Christodoulou, G.N. 2008. A 6-month study of postpartum depression and related factors in Athens Greece. Comprehensive Psychiatry. 49(3):27582.

- Holcomb, W.L., Stone, L.S., Lustman, P.J., Gavard, J.A, Mostello, D.J. 1996. Screening for depression in pregnancy: characteristics of the Beck Depression Inventory. Obstetrics and Gynecology. 88:1021-5.

- Honikman, s., van Heyingen, T., Field, S., Baron, E., Tomlinson, M. 2012. Stepped Care for Maternal Health: A Case Study of the Perinatal Mental Health Project in South Africa. PLoS Med. 9(5): e 1001222. https://doi.org/10.1371/journal.pmed. 1001222.

- Kaaya, S.F., Mbwambo, J.K., Kilonzo, G., van den Borne, H., Leshabari, M.T., Fawzi, M.C., et al. Socioeconomic and partner relationship factors associated with antenatal depressive morbidity among pregnant women in Dar es Salaam, Tanzania. Tanzanian Journal of Health Research. 12(1):23-35.

- Kathree, T. Selohilwe, O.M., Bhana, A. Petersen, I. 2014. Perceptions of postnatal depression and health care needs in a south African sample: the "mental" in maternal health care. BMC Women's Health.14: 140. http://www.biomedcentral.com/1472-6874/14/140.

- Kendell, R.E., Chalmers, K.C., Platz, C. 1987. Epidemiology of puerperal psychoses. British Journal of Psychiatry. 150:662-73.

- Marsay, C. Manderson, L., Subramaney, U. 2017. Validation of the Whooley questions for antenatal depression and anxiety among loe-income women in urban South Africa. S Afr J Psychiat. 23(0), a1013. https://doi.org/10.4102/sajpsychiatry.v23i0.1013

- Mota, N., Cox, B.J., Enns, M.W., Calhoun, C. Sareen, J. 2008. The relationship between mental disorders, quality of life, and pregnancy: findings from a nationally representative sample. Journal of Affective Disorders. 109:300-4.

- Munk-Olsen, T., Laursen, T.M., Pedersen, C.B., Mors, O., Mortensen, P.B. 2006. New parents and mental disorders: a population-based register study. JAMA. 296:2582-9.

- Najman, J.M., Andersen, M.J., Bor, W., O'Callaghan M.J., Williams G.M. 2000. Postnatal depressionmyth and reality: maternal depression before and after the birth of a child. Social Psychiatry and Psychiatric Epidemiology. 35:19-27.

- O'Hara, M.W., Swain, A.M. 1996. Rates and risks of postpartum depression: a meta- analysis. International Review of Psychiatry. 8:37-54.

- Patel V. 2007. Mental health in low and middle-income countries. 81:81-96.

- Porcels, J., Feigal, C., Poe, L., Postma, I.R., Zeeman, G.G., Olowoyey, A. et al 2013. Hypertensive disorder of pregnancy and risk of screening positive for posttraumatic stress disorder: a cross- 
sectional study. Pregnancy Hypertension. International Journal of Women's Cardiovascular Health. 3:227-234.

- Postma, I., Wessel, I., Aarnoudse, J., Zeeman, G. 2010. Neurocognitive functioning in women with a history of eclampsia: executive functioning and sustained attention. American Journal of Perinatology. 27(9):685-90.

- Postma, I.R., Groen, H., Eastering, F.R., Tsigas, E.Z., Wilson, M.L. 2013. The brain study. Cognition, quality of life and social functioning following preeclampsia: an observational study. Pregnancy Hypertension. International Journal of Women's Cardiovascular Health. 3:227-34.

- Rahman, A., Creed, F. 2010. Outcome of prenatal depression and risk factors associated with persistence in first postnatal year: prospective study from Rawalpindi, Pakistan. Journal of Affective Disorders. 100:115-21.

- Ronsmans, C., 2009. Severe acute maternal morbidity in low-income countries. Best practice \& research Clinical obstetrics \& gynaecology, 23(3): 305-316.

- Ronsmans, C., Graham, W.J. 2006. Maternal mortality: who, when, where, and why. 368:1189-1200.

- Senturk, V., Hanlon, C., Medhin G., Dewey, M., Araya, M., Alem, A. et al. Impact of perinatal somatic and common mental disorder symptoms on functioning in Ethiopian women: The P-MaMiE populationbased cohort study. Journal of Affective Disorders. 136:340-9.

- Simon, G.E., VonKorff, M., Piccinelli, M., Fullerton, C., Ormel, J. 1999. An international study of the relation between somatic symptoms and depression. New England Journal of Medicine. 341(18):1329-35.

- Souza, J.P., Cecatti, J.G., Parpinelli, M.A., Krupa, F., Osis, M.J.D. 2009. An emerging "maternal nearmiss syndrome": narratives of women who almost died during pregnancy and childbirth. 36(2):14958.

- Sword, W., Landy, C.K., Thabane, L., Watt, S., Krueger, P., Farine, D. et al. 2011. Is mode of delivery associated with postpartum depression at 6 weeks: a prospective cohort study. British Journal of Obstetrics and Gynaecology. 118(8):966-77.

- Weeks, A., Lavender, T., Nazziwa, E., Mirembe, F. 2005. Personal accounts of 'near-miss' maternal mortalities in Kampala, Uganda. British Journal of Obstetrics and Gynaecology. 112:1302-7.

- World Health Organization. [Internet]. 2008. The Global Burden of Disease (GBI) study: projections of mortality and burden of disease 2004 - 2030. [cited 2016 Mar]. Available from : http://www.who.int/healthinfo/global_burden_disease/projections2004/en/

- World Health Organization. 2004b. Beyond the numbers: reviewing maternal deaths and complications to make pregnancy safe. Geneva: World Health Organization. 